\title{
La communication du cancer
}

\section{Cancer Communication}

\section{P. Guex}

(C) Lavoisier SAS 2014

Depuis un quart de siècle, la question de la communication en oncologie a été l'objet d'un large intérêt dans les domaines de la recherche, de la formation et de l'enseignement, notamment dans les pays anglo-saxons, en Suisse et en Belgique. Il s'agit des formations aux aptitudes à la communication, les communications skills trainings (CSTs) selon la terminologie internationale consacrée.

Les états généraux de la Ligue contre le cancer en 1998 ont permis de faire émerger les demandes des patients concernant le moment clé de l'annonce du diagnostic de cancer. Expérimenté entre 2004 et 2005, cela fera dix ans qu'en France le dispositif d'annonce et les infirmières qui l'animent se sont progressivement installés en clinique à la faveur des trois Plans cancer.

La Société Française de Psycho-Oncologie (SFPO), lors de son congrès en 2013, a consacré un atelier à la présentation de différentes formes de formation ou d'entraînement à la communication médecin-malade, soignant-soigné, dont sont issues certaines contributions de ce dossier.

La SFPO a également lancé un « tour de France » de sensibilisation à l'alliance thérapeutique et à la communication avec les patients en oncologie, sous la forme d'ateliers organisés à Paris et dans différentes régions, au cours desquels des comédiens jouent des scénettes mettant en scène des consultations avec des personnes atteintes de cancer. À partir de ces ateliers, les participants (pour le moment majoritairement des médecins) sont conduits à réfléchir à leurs pratiques et à animer des jeux de rôles mettant en scène leurs propres attitudes et émotions.

Les articles publiés dans ce dossier illustrent bien les diverses facettes de la problématique rencontrée à chacun des ateliers : la communication est-elle standardisable, modélisable, optimisable à l'image des traitements en oncologie, afin d'arriver rapidement et magiquement à l'acceptation d'un programme personnalisé de soins avec le patient et ses proches ? Dans ce cas est-ce par la simulation avec des

P. Guex $(\bowtie)$

CHUV, faculté de biologie et médecine,

1011 Lausanne, Suisse

e-mail : Patrice.Guex@chuv.ch acteurs, la réassurance, la négociation, la gestion des émotions qu' on y arrive le mieux ? Est-ce un modèle purement pédagogique qu'il faut développer ? Est-ce approprié de créer un environnement standard, mais adaptable, conçu pour toutes les situations ? On sait malheureusement que cela peut entretenir nombre de clichés, notamment lors de la désignation des émotions, qui passent radicalement à côté d'une écoute authentique du vécu individuel des patients, en se focalisant essentiellement sur leurs besoins et les actions à entreprendre.

Comment prendre en compte le traumatisme de l'annonce et l'angoisse qu'elle génère, jusqu'à quel degré peut-on se laisser interpeller par la souffrance d'autrui ? Peut-on rester scientifique, hypothéticodéductif d'un côté et trouver en même temps en soi assez de disponibilité pour accueillir les paroles et les silences de la personne, sans aussitôt les couvrir de sa propre volubilité, de son inquiétude et de sa mise en œuvre de solutions immédiates ? Peut-on accepter, durant les tours de paroles entre soignants, médecin et soignés, que des phases rationnelles, explicatives, alternent avec des moments où, au-delà du simple désir de savoir, les patients s'interrogent sur la signification ou le sens de ce qui leur arrive, sur des sujets sans réponse auxquels ils ne peuvent s'empêcher de penser (destin, mort, justice), sur la gamme des sentiments - insécurité, incrédulité, abandon, hostilité qui surviennent en écho aux émotions qu'ils ressentent — tristesse, culpabilité, honte, colère — sans qu'aussitôt chaque intervenant les rassure ? Comment rendre vraiment à la communication son rôle de construction de significations communes qui serviront de référence pour la suite des échanges, et quelles sont les conditions de sa réussite?

Pour compléter la richesse de contenu de ce numéro, nous avons demandé à Pascal Singy, professeur de sociolinguistique à l'université et en médecine, qui mène entre autres, avec l'équipe de Lausanne du Pr F. Stiefel, une recherche sur la migration et la communication interculturelle, de rédiger un commentaire sur la problématique de la parole du soignant et du soigné dans un monde et des institutions médicales plurilingues et pluriculturelles ; celles-ci requièrent dorénavant de véritables compétences cliniques transculturelles. 